\title{
DISTRIBUIÇÃO E CARACTERIZAÇÃO DE SUBSTÂNCIAS HÚMICAS EM VERMICOMPOSTOS DE ORIGEM ANIMAL E VEGETAL $^{(1)}$
}

\author{
Rosa Maria Vargas Castilhos ${ }^{(2)}$, Deborah Pinheiro Dick ${ }^{(3)}$, Danilo Dufech Castilhos ${ }^{(2)}$, \\ Tânia Beatriz Araújo Gamboa Morselli( ${ }^{(2)}$, Paula Fernanda Pinto da Costa ${ }^{(4)}$, Wagner \\ Bertuol Casagrande ${ }^{(5)} \&$ Carla Machado da Rosa ${ }^{(6)}$
}

\begin{abstract}
RESUMO
A utilização de resíduos orgânicos, como fertilizantes e condicionadores de solo, requer sua maturação e monitoramento da qualidade do material resultante. A vermicompostagem é uma técnica que, ao longo do processo, elimina os potenciais efeitos adversos dos resíduos à saúde humana e ao solo. A avaliação das substâncias húmicas $(\mathrm{SH})$ nos produtos finais quanto à quantidade e à qualidade permite inferir sobre o grau de estabilidade e maturidade dos vermicompostos. Este trabalho objetivou avaliar a qualidade de seis vermicompostos das seguintes matériasprimas: esterco bovino (EB), esterco ovino (EO), esterco suíno (ES), esterco de codorna (EC), borra de café (BC) e de erva-mate (BE) com respeito ao teor de ácidos húmicos $(\mathrm{AH})$ e de ácidos fúlvicos (AF) e às suas características químicas, após 70 dias de compostagem. O fracionamento químico da matéria orgânica foi realizado com base na solubilidade em meio básico e ácido e a distribuição de cada fração calculada como percentual do $\mathrm{C}$ total. Foram calculados os índices de humificação: percentual de $\mathrm{AH}$ e razão $\mathrm{AH} / \mathrm{AF}$. Nos $\mathrm{AH}$ e $\mathrm{AF}$ purificados, determinaram-se a composição elementar (CHNO), a composição química por espectroscopia de infravermelho com transformada de Fourier (FTIR) e o índice de aromaticidade $\mathrm{I}_{1630} / \mathrm{I}_{2920}$. $\mathrm{O}$ teor de substâncias húmicas $(\mathrm{AH}+\mathrm{AF})$ decresceu na seqüência: $\mathrm{BC}>\mathrm{EO} \approx \mathrm{BE} \approx \mathrm{EB}>\mathrm{ES}>\mathrm{EC}$, tendo sua composição química também diferido entre os vermicompostos. O grau de maturidade foi superior nos
\end{abstract}

\footnotetext{
(1) Trabalho apresentado no XXXI Congresso Brasileiro de Ciência do Solo, 2007 (Gramado, RS).

(2) Professor Associado do Departamento de Solos, Faculdade de Agronomia Eliseu Maciel, Universidade Federal de Pelotas (UFPEL). Caixa Postal 354. CEP 96010-900 Pelotas (RS) E-mail: rosamvc@ufpel.edu.br

(3) Professor Associado do Instituto de Química, Universidade Federal do Rio Grande do Sul (UFRGS). Av.Bento Gonçalves 9500. CEP 91501-907 Porto Alegre (RS). Bolsista CNPq. E-mail: dpdick@iq.ufrgs.br

(4) Estudante do curso de Agronomia, UFPEL, Bolsista de Iniciação Científica CNPq. E-mail: costapfp@yahoo.com.br

(5) Estudante do Curso de Engenharia Química, UFRGS. Bolsista de Iniciação Científica da FAPERGS. E-mail: wagnercasa@hotmail.com

(6) Doutoranda do Programa Pós-graduação em Agronomia, Solos, UFPEL. E-mail: carlamrosa@yahoo.com.br
} 
vermicompostos de resíduos de origem vegetal (borra de café e de erva-mate). Os AH desses dois vermicompostos apresentaram menor proporção de grupos oxigenados e menor caráter aromático.

Termos de indexação: grau de humificação, ácidos fúlvicos, ácidos húmicos, resíduos orgânicos.

\title{
SUMMARY: DISTRIBUTION AND CHARACTERIZATION OF HUMIC SUBSTANCES IN ANIMAL AND PLANT VERMICOMPOST
}

\begin{abstract}
The use of organic residues as fertilizers and soil conditioners requires their maturation and the quality monitoring of the final product. Vermicomposting is a technique which, along with the composting process, eliminates the potential harmful effect of manure residues to human health and soil. The stability and maturity degrees of a given vermicompost are usually inferred from the quantity and quality of the humic substances in the resulting compost. This study aimed to evaluate the quality and maturity of vermicomposts from six different residues: cattle manure $(C M)$, sheep manure $(S M)$, pig manure $(P M)$, quail manure $(Q M)$, coffee dregs $(C D)$ and mate-tee dregs $(M D)$, by determining the content of humic substances (humic, HA, and fulvic acids (FA) and their chemical composition, after 70 days of composting. The humic substances were chemically fractionated according to their solubility in basic and acidic medium. The distribution of total $C$ in the different humic fractions was determined, and the humification indices $H A$ percentage and $H A / F A$ ratio were calculated. The elemental composition (CHNO) and chemical composition by infrared spectroscopy (IRSP) were determined in the purified HA and FA. An aromaticity index $\left(I 1_{630} / I_{2920}\right)$ was calculated based on the IRSP spectra. The vermicomposts differed in humic substance content $(F A+H A)$, which decreased in the order $C D>S M \approx M D \approx C M>P M>Q M$. The maturity degree was greater in the vermicomposts of vegetal residues (coffee and mate-tee dregs). The HA showed that the proportion of $O$ containing functional groups and the aromatic degree were lowest in these two vermicomposts.
\end{abstract}

Index terms: humification degree, fulvic acids, humic acids, organic residues.

\section{INTRODUÇÃO}

A necessidade de preservar o meio ambiente tem estimulado o aproveitamento, como fertilizantes e, ou, condicionadores de solo, dos mais variados tipos de resíduos orgânicos, gerados em atividades rurais, agroindustriais ou urbanas, proporcionando também retornos econômicos e melhoria na qualidade do solo (Tedesco et al., 1999). Alguns resíduos necessitam de uma prévia decomposição antes de serem incorporados ao solo, a fim de reduzir possíveis efeitos adversos à saúde humana, às plantas e ao solo. Dentre estes efeitos, citam-se: a contaminação com organismos patogênicos (fungos, bactérias, vírus e helmintos), a imobilização de $\mathrm{N}$ decorrente da elevada relação $\mathrm{C} / \mathrm{N}$ de alguns materiais, as possíveis alterações no $\mathrm{pH}$ do solo decorrentes da elevada acidez ou alcalinidade dos resíduos.

A vermicompostagem é uma técnica de decomposição que utiliza minhocas, requer pouco consumo de energia e fornece um material estabilizado, principalmente quanto a $\mathrm{pH}$, relação $\mathrm{C} / \mathrm{N}$, em tempo menor, se comparado com a compostagem (Tibau, 1984).
A região Sul do Estado do Rio Grande do Sul caracteriza-se por intensa atividade agropastoril, em que a pecuária extensiva bovina e ovina tem papel destacado e por pequenas propriedades, onde, além da bovinocultura de leite, há criação de suínos e aves. Também é tradição na região o consumo de café e chimarrão. Essas atividades geram resíduos orgânicos que podem ser aproveitados como matéria-prima para vermicompostagem, obtendo-se produtos finais com distintas características químicas.

A avaliação das substâncias húmicas (SH), quanto à quantidade de ácidos húmicos $(\mathrm{AH})$ e fúlvicos $(\mathrm{AF}) \mathrm{e}$ sua qualidade permite inferir sobre o grau de estabilidade e maturidade da matéria orgânica presentes nos produtos finais (Senesi et al., 1996). O fracionamento químico da matéria orgânica permite a separação e quantificação destas frações, que, após serem purificadas, podem ser caracterizadas quanto à composição química e quanto ao aspecto molecular (Guerra \& Santos, 1999). A espectroscopia de infravermelho permite identificar os grupos reacionais e os componentes estruturais nas substâncias húmicas (Ceretta et al.,1999). Em geral, 
conforme progride a humificação na compostagem ocorre aumento do teor de substâncias húmicas solúveis em meio alcalino (AH e AF), sendo os índices de humificação e de maturação do composto calculados de acordo com os teores dessas substâncias (Chen \& Inbar, 1993; Chefetz et al, 1996).

Estudos realizados sobre a compostagem de resíduos agroindustriais (Antunes et al., 2005), resíduos sólidos urbanos (Chen \& Inbar, 1993; Chefetz et al, 1996) e sobre a decomposição de lodos orgânicos, oriundos de fabricação de papel (Dick et al., 1997), mostraram que esses processos tendem a reduzir os teores totais de $\mathrm{C}(\mathrm{COT})$ e a proporção da fração ácidos fúlvicos (AF), com aumento da fração ácido húmicos $(\mathrm{AH})$, refletindo-se no aumento do seu grau de maturidade. Em AH de resíduos agroindustriais compostados por 150 dias, Antunes (2005) constatou aumento no teor de N, decréscimos no de $\mathrm{O}$ e na relação $\mathrm{C} / \mathrm{N}$ e constância nos teores de $\mathrm{C}$ e H. No caso específico de vermicompostos, Leal et al. (2006) observaram diferenças entre cinco compostos de resíduos agroindustriais após 89 dias de vermicompostagem: os teores de COT e de $\mathrm{SH}$ e o grau de humificação foram maiores em compostos de origem vegetal comparados com os de origem animal.

Visando à possibilidade de dar um destino agronômico a resíduos agroindustriais produzidos na região de Pelotas, RS, este trabalho objetivou avaliar a qualidade de seis vermicompostos de resíduos de origem animal e vegetal com respeito ao teor de ácidos húmicos (AH) e de ácidos fúlvicos (AF) e as suas características químicas e moleculares.

\section{MATERIAL E MÉTODOS}

\section{Local e origem dos vermicompostos}

Foram estudadas amostras de vermicompostos de resíduos orgânicos de origem animal - esterco bovino (EB), esterco ovino (EO), esterco suíno (ES) e esterco de codorna (EC) e vegetal - borra de café (BC) e de erva-mate (BE). A vermicompostagem foi feita por Huber (2003) num experimento do Departamento de Solos da Faculdade de Agronomia da UFPEL, no ano de 2005 , em caixas de madeira, utilizando minhocas da espécie Eisenia foetida. Os tratamentos foram arranjados em blocos ao acaso, com quatro repetições. Ao final do processo (70 dias), os vermicompostos foram peneirados e secos em estufa a $65^{\circ} \mathrm{C}$ até peso constante e moídos em gral para as determinações deste trabalho. Os resíduos de EB, ES e EC foram provenientes da Fazenda Experimental da UFPEL, Pelotas, o de EO da EMBRAPA-CPPSUL, Bagé. A erva-mate (Madrugada $\left.{ }^{\circledR}\right)$ é produzida no RS e a borra de café foi obtida junto às cafeterias do município de Pelotas.

\section{Fracionamento, quantificação e purificação de SH}

O fracionamento químico da matéria orgânica em $\mathrm{AH}$ e AF foi feito com base na solubilidade em meio ácido e básico (Dick et al., 1997). A 1 g de vermicomposto adicionaram-se $30 \mathrm{~mL}$ de $\mathrm{HCl} 0,5$ mol L-1, agitando-se a suspensão por $2 \mathrm{~h}$. O sobrenadante que continha a fração não-húmica (SNH) foi separada por centrifugação (15 min a $2500 \mathrm{rpm}$ ), e o volume total foi medido. Ao resíduo sólido adicionaram-se $30 \mathrm{~mL}$ de $\mathrm{NaOH} 0,5 \mathrm{~mol} \mathrm{~L}^{-1}$, procedeu-se a agitação por três horas e centrifugação (15 min a $2500 \mathrm{rpm}$ ), repetindo-se tal procedimento até o sobrenadante ficar claro. Em cada extração, o sobrenadante que continha as substâncias húmicas solúveis $(\mathrm{SH}=\mathrm{AH}+\mathrm{AF})$ foi transferido para um frasco coletor, e o volume final foi completado a $200 \mathrm{~mL}$. Retirou-se uma alíquota de $20 \mathrm{~mL}$ para análise de $\mathrm{C}$, e o restante foi acidificado com $\mathrm{HCl} 0,5 \mathrm{~mol} \mathrm{~L}^{-1}$ até $\mathrm{pH} 2,0$. Após $24 \mathrm{~h}$ de repouso, o $\mathrm{AH}$, insolúvel em $\mathrm{pH}$ ácido, foi separado por centrifugação do AF, solúvel. O teor de $\mathrm{C}$ nos extratos de $\mathrm{SNH}, \mathrm{AF}$ e $\mathrm{SH}$ foi quantificado espectroscopicamente, medindo-se a absorbância a $580 \mathrm{~nm}$, após reação com solução ácida de dicromato de potássio $0,625 \mathrm{~mol} \mathrm{~L}^{-1}$, durante $4 \mathrm{~h}$, a $60{ }^{\circ} \mathrm{C}$, empregando-se uma curva padrão de $\mathrm{D}$ glucose anidra de 0 a $200 \mathrm{mg} \mathrm{L}^{-1}$ de C.

$\mathrm{O} \mathrm{C}$ contido na forma de $\mathrm{AH}\left(\mathrm{C}_{\mathrm{AH}}\right)$ foi estimado por diferença entre o $\mathrm{C}$ do extrato de $\mathrm{SH}\left(\mathrm{C}_{\mathrm{SH}}\right)$ e o $\mathrm{C}$ do extrato de $\mathrm{AF}\left(\mathrm{C}_{\mathrm{AF}}\right)$. Foram calculados a distribuição de cada fração como percentual do $\mathrm{C}$ orgânico total e os índices de humificação: percentual de ácidos húmicos $\left(\mathrm{HP}=\mathrm{C}_{\mathrm{AH}} / \mathrm{C}_{\mathrm{SH}} \cdot 100\right)$ e razão de humificação ( $\left.\mathrm{HR}=\mathrm{C}_{\mathrm{AH}} / \mathrm{C}_{\mathrm{AF}}\right)$, segundo Chefetz et al. (1996).

As amostras de $\mathrm{AH}$ foram purificadas com solução de $\mathrm{HCl}+\mathrm{HF} 5 \% / 5 \%$ (v/v) e secas em estufa a vácuo a $60{ }^{\circ} \mathrm{C}$. Os AF foram purificados por tratamento em resina XAD-8, seguido de tratamento com resina Amberlite IR-120 (Dick et al.,1997). Amostras de AF purificadas foram secas em estufa a vácuo a $60^{\circ} \mathrm{C}$.

\section{Análise elementar}

O teor de C orgânico total (COT) nos vermicompostos foi determinado pelo método WalkleyBlack, com calor externo (Tedesco et al., 1995). Nas amostras purificadas de $\mathrm{AH}$ e $\mathrm{AF}$ e nos vermicompostos inteiros, foi determinada a composição elementar (CHN) (Perkin Elmer, 2400) e o teor de O nos AH e AF foi calculado por diferença de $1000 \mathrm{~g} \mathrm{~kg}^{-1}$, após correção dos valores para uma base livre de cinzas. $\mathrm{O}$ teor de cinzas foi determinado gravimetricamente, após calcinação a $750{ }^{\circ} \mathrm{C}$ durante $4 \mathrm{~h}$. A partir dos resultados, foram calculadas a relação $\mathrm{C} / \mathrm{N}$ e as razões atômicas $\mathrm{H} / \mathrm{C}$ e $\mathrm{O} / \mathrm{C}$. 


\section{Espectroscopia de infravermelho}

As amostras purificadas de $\mathrm{AH}$ e $\mathrm{AF}$ e os vermicompostos foram analisados por espectroscopia de infravermelho com transformada de Fourier (FTIR), em pastilhas de KBr (Shimadzu 830FTIR) na proporção amostra: $\mathrm{KBr}$ de 1:100. Previamente à análise, as amostras foram secas em estufa sob vácuo a $60^{\circ} \mathrm{C}$ durante $24 \mathrm{~h}$. Os espectros de infraver-melho foram interpretados segundo Dick et al. (2003). A partir dos dados de FTIR (análises em duplicata), foi calculado o índice de aromaticidade $\mathrm{I}_{1630} / \mathrm{I}_{2920}$ (Chefetz et al., 1996), dividindo-se a intensidade de absorção em torno de $1630 \mathrm{~cm}^{-1}$ pela intensidade a $2922 \mathrm{~cm}^{-1}$ apenas para os AH e AF dada a interferência das bandas da fração inorgânica nos espectros dos vermicompostos. O valor da intensidade foi obtido utilizando o software do aparelho, após ajustar-se a linha de base geral do espectro em três a quatro pontos no mesmo (aproximadamente em 3800, 1750, 980 e $500 \mathrm{~cm}^{-1}$ ). Para a medição da intensidade dos grupos aromáticos, estabeleceu-se uma linha de base entre 1696 e $1530 \mathrm{~cm}^{-1}$ e para os grupos alifáticos, entre 3000 e $2800 \mathrm{~cm}^{-1}$.

\section{Análise estatística}

Os resultados referentes aos teores de COT e na forma de substâncias húmicas $\left(\mathrm{C}_{\mathrm{SH}}\right)$, ácidos húmicos $\left(\mathrm{C}_{\mathrm{AH}}\right)$ e ácidos fúlvicos $\left(\mathrm{C}_{\mathrm{AF}}\right)$ foram submetidos à análise de variância e as médias foram comparadas pelo teste de Duncan, a $5 \%$. Os resultados da composição elementar e a espectroscopia de infravermelho dos vermicompostos inteiros e dos ácidos húmicos e fúlvicos foram obtidos em amostras compostas das quatro repetições e, por esta razão, não foram analisados estatisticamente.

\section{RESULTADOS E DISCUSSÃO}

\section{Composição elementar dos vermicompostos e distribuição de substâncias húmicas}

O conteúdo de COT variou de $232 \mathrm{~g} \mathrm{~kg}^{-1}$ a $457 \mathrm{~g}$ $\mathrm{kg}^{-1}$, sendo inferior nos vermicompostos de resíduos de origem animal, com exceção do EC (Quadro1). Esse vermicomposto apresentou o menor teor de $\mathrm{SH}$ $(\mathrm{AH}+\mathrm{AF})$ do grupo estudado o que pode estar relacionado com a composição do resíduo original: após a vermicompostagem, fragmentos de serragem, que é um material lignítico usado como cama para as codornas, eram ainda visíveis. O maior teor absoluto de substâncias húmicas solúveis foi observado no BC. Esse vermicomposto também apresentou a maior proporção de $\mathrm{C}$ na forma de substâncias húmicas solúveis (76 \%) (Figura 1). O vermicomposto EC apresentou a menor proporção de $\mathrm{C}_{\mathrm{SH}}(7 \%)$ entre o grupo estudado, enquanto nas outras amostras, a proporção de $\mathrm{SH}$ variou de 32 a 58 \%. Em todas as amostras, a proporção de $\mathrm{C}_{\mathrm{AH}}(14$ a $55 \%)$ foi superior à de $\mathrm{C}_{\mathrm{AF}}(6 \mathrm{a} 21 \%)$.

Em geral, a compostagem é acompanhada pelo aumento da razão de humificação $\left(\mathrm{HR}=\mathrm{C}_{\mathrm{AH}} / \mathrm{C}_{\mathrm{AF}}\right)$ e do percentual de ácidos húmicos $\left(\mathrm{HP}=\mathrm{C}_{\mathrm{AH}} / \mathrm{C}_{\mathrm{SH}} .100\right)$ (Chefetz et al., 1996). Os vermicompostos de origem vegetal (BC e BE) apresentaram maior $\mathrm{HR}$ e $\mathrm{HP}$ (Quadro1), indicando grau de maturação mais elevado nesses dois materiais. Esses valores aproximaram-se dos encontrados por Chefetz et al. (1996), que, após 132 dias de compostagem de resíduos sólidos urbanos, obtiveram valor de 2,9 para HR e de 75 para HP e por Castilhos et al. (2007), que, após 90 dias de vermicompostagem de resíduos de origem vegetal, atingiram índices $\mathrm{HR}$ de 2,6 e 3,1 e HP de 72 e 75. Já Antunes (2005) encontrou valores superiores para compostos de resíduos da agroindústria após 150 dias de compostagem, que se situaram entre 6,5 e 8,3 para HR e acima de 80 para HP. A razão $\mathrm{C} / \mathrm{N}$ dos vermicompostos variou de 5,8 a 7,9 sendo os maiores valores observados nas amostras de origem vegetal. Esse comportamento decorre provavelmente do menor teor de material protéico em resíduos vegetais em comparação ao de animais. Nessas amostras, BC e BE, a razão $\mathrm{H} / \mathrm{C}$ foi de 1,0 e 0,92, respectivamente. Com exceção da amostra EC, a razão H/C nas amostras de origem animal foi $=1,0$, indicando um caráter mais alifático do que as de origem vegetal. $\mathrm{O}$ valor de 0,36 observado para a do vermicomposto EC pode estar relacionado com a presença de fragmentos de serragem. Esse material, por conter lignina, apresenta comparativamente maior abundância de grupos aromáticos (Stevenson, 1994), o que leva a um baixo valor para a razão $\mathrm{H} / \mathrm{C}$.

Quadro 1. Teor de carbono orgânico total (COT) e carbono na forma de substâncias húmicas $\left(\mathrm{C}_{\mathrm{SH}}\right)$, ácidos húmicos $\left(\mathrm{C}_{\mathrm{AH}}\right)$ e ácidos fúlvicos $\left(\mathrm{C}_{\mathrm{AF}}\right)$, razão de humificação (HR), percentual de ácidos húmicos $(\mathrm{HP})$, razão $\mathrm{C} / \mathrm{N}$ e razão atômica $\mathrm{H} / \mathrm{C}$ da matéria orgânica de seis vermicompostos

\begin{tabular}{lcccccc}
\hline & E B & BC & EC & BE & EO & ES \\
\hline COT $\left(\mathrm{g} \mathrm{kg}^{-1}\right)$ & $326 \mathrm{~d}$ & $457 \mathrm{a}$ & $413 \mathrm{~b}$ & $374 \mathrm{c}$ & $269 \mathrm{e}$ & $232 \mathrm{f}$ \\
$\mathrm{C}_{\mathrm{SH}}\left(\mathrm{g} \mathrm{kg}^{-1}\right)$ & $132 \mathrm{~b}$ & $348 \mathrm{a}$ & $32 \mathrm{~d}$ & $144 \mathrm{~b}$ & $146 \mathrm{~b}$ & $76 \mathrm{c}$ \\
$\mathrm{C}_{\mathrm{AH}}\left(\mathrm{g} \mathrm{kg}^{-1}\right)$ & $67 \mathrm{c}$ & $252 \mathrm{a}$ & $6,5 \mathrm{e}$ & $104 \mathrm{~b}$ & $94 \mathrm{~b}$ & $33,5 \mathrm{~d}$ \\
$\mathrm{C}_{\mathrm{AF}}\left(\mathrm{g} \mathrm{kg}^{-1}\right)$ & $65 \mathrm{~b}$ & $96,6 \mathrm{a}$ & $25,2 \mathrm{~d}$ & $40,2 \mathrm{c}$ & $52 \mathrm{~b}$ & $42,3 \mathrm{c}$ \\
$\mathrm{HR}$ & 1,02 & 2,61 & 0,26 & 2,57 & 1,81 & 0,79 \\
$\mathrm{HP}$ & 51 & 72 & 21 & 72 & 64 & 44 \\
$\mathrm{C} / \mathrm{N}$ & 6,8 & 7,9 & 7,3 & 7,5 & 6,2 & 5,8 \\
$\mathrm{H} / \mathrm{C}$ & 1,1 & 1,0 & 0,36 & 0,92 & 1,2 & 1,0 \\
\hline
\end{tabular}

EB: esterco bovino; BC: borra de café; EC: esterco de codorna; BE: borra de erva-mate; EO: esterco ovino; ES: esterco suíno. Médias seguidas pela mesma letra, na linha, não diferem pelo Teste de Duncan a $5 \%$. 


\section{$\square$ FINS $\square \mathrm{SNH} \square \mathrm{AH} \quad \square \mathrm{AF}$}

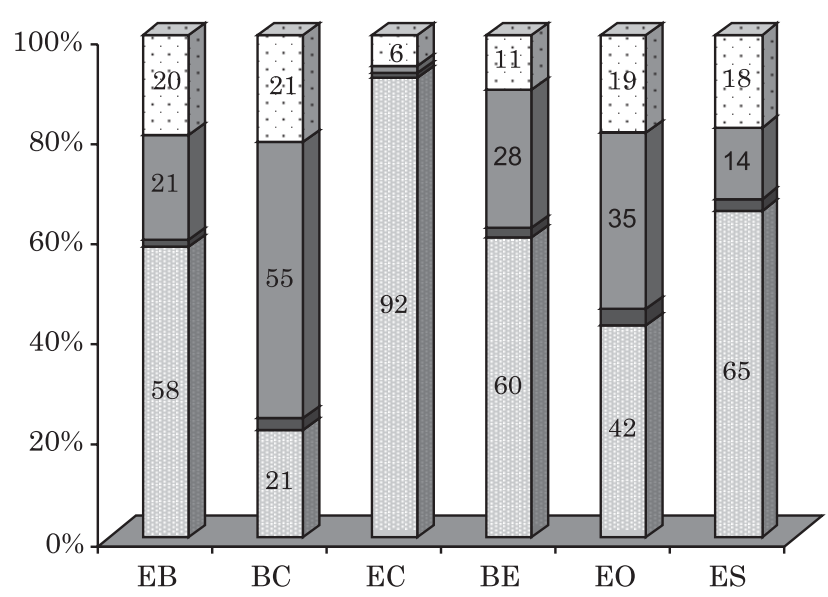

Figura 1. Distribuição das frações da matéria orgânica: fração insolúvel (FINS) substâncias não-húmicas (SNH), ácidos húmicos (AH) e ácidos fúlvicos (AF), como percentual do carbono total em diferentes vermicompostos. EB: esterco bovino; BC: borra de café; EC: esterco de codorna; BE: borra de erva-mate; EO: esterco ovino; ES: esterco suíno.

\section{Composição elementar das substâncias húmicas}

$\mathrm{O}$ teor de cinzas nos $\mathrm{AH}$ foi inferior a $1 \%$, enquanto nos $\mathrm{AF}$ variou de 12 a $84 \%$ (dados não mostrados). Os $\mathrm{AH}$ em média apresentaram teores mais elevados de $\mathrm{C}$ e de $\mathrm{N}$, e menores valores de $\mathrm{O}$ do que $\mathrm{AF}$ (Quadro 2). Essas diferenças refletem-se nos valores da razão $\mathrm{C} / \mathrm{N}$ e da razão $\mathrm{O} / \mathrm{C}$ : $\mathrm{AF}$ são mais oxigenados e menos nitrogenados do que seus respectivos AH. Em comparação a amostras de solos, a composição química dos $\mathrm{AH}$ foi semelhante à observada em $\mathrm{AH}$ pedogênicos (Dick et al., 2003), com exceção do teor de $\mathrm{N}$ que foi em média mais elevado. A elevada razão $\mathrm{O} / \mathrm{C}$, observada nos $\operatorname{AF}(0,98$ a 1,43), indica serem essas amostras mais ricas em grupos oxigenados do que as usualmente encontradas em solos (Stevenson,1994).

Tanto os AH como os AF apresentaram relação $\mathrm{C} /$ $\mathrm{N}$ e razão $\mathrm{H} / \mathrm{C}$ superiores às determinadas nos respectivos vermicompostos (Quadros 1 e 2). Infere-se que a fração insolúvel seja mais rica em $\mathrm{N}$ e contenha mais estruturas aromáticas em sua composição do que as substâncias húmicas.

\section{Espectroscopia de infravermelho (FTIR)}

As amostras de $\mathrm{AH}$ e $\mathrm{AF}$ apresentaram o mesmo padrão de espectro de FTIR (Figuras 2), em que os principais grupos funcionais e respectivas regiões de absorção foram identificados: estiramento de-OH em ponte $\left(3400 \mathrm{~cm}^{-1}\right)$; estiramento de $\mathrm{C}-\mathrm{H}$ alifático $(2920$ e $\left.2850 \mathrm{~cm}^{-1}\right)$, estiramento de $\mathrm{C}=\mathrm{O}$ de grupos $-\mathrm{COOH}$

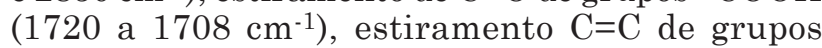

aromáticos (1650 a $\left.1630 \mathrm{~cm}^{-1}\right)$, estiramento $\mathrm{C}-\mathrm{N}-\mathrm{H}$ de amidas (1530 a $\left.1507 \mathrm{~cm}^{-1}\right)$, estiramento de $\mathrm{C}-\mathrm{O}$ de ácidos carboxílicos $\left(1226 \mathrm{~cm}^{-1}\right)$, estiramento $\mathrm{C}-\mathrm{O}$ de polissacarídeos ( de 1120 a $1090 \mathrm{~cm}^{-1}$ ), absorção de SiO e outras ligações em silicatos na região de $1031 \mathrm{~cm}^{-1}$ (Dick et al., 2003). Nas amostras de AF, as bandas de absorção em torno de 1720 e de $1100 \mathrm{~cm}^{-1}$ foram proporcionalmente mais intensas do que nos respectivos $\mathrm{AH}$. A maior proporção de grupos carboxílicos e de polissacarídeos nos AF é confirmada pelos valores elevados de razão $\mathrm{O} / \mathrm{C}$, determinados nos mesmos (Quadro 2).
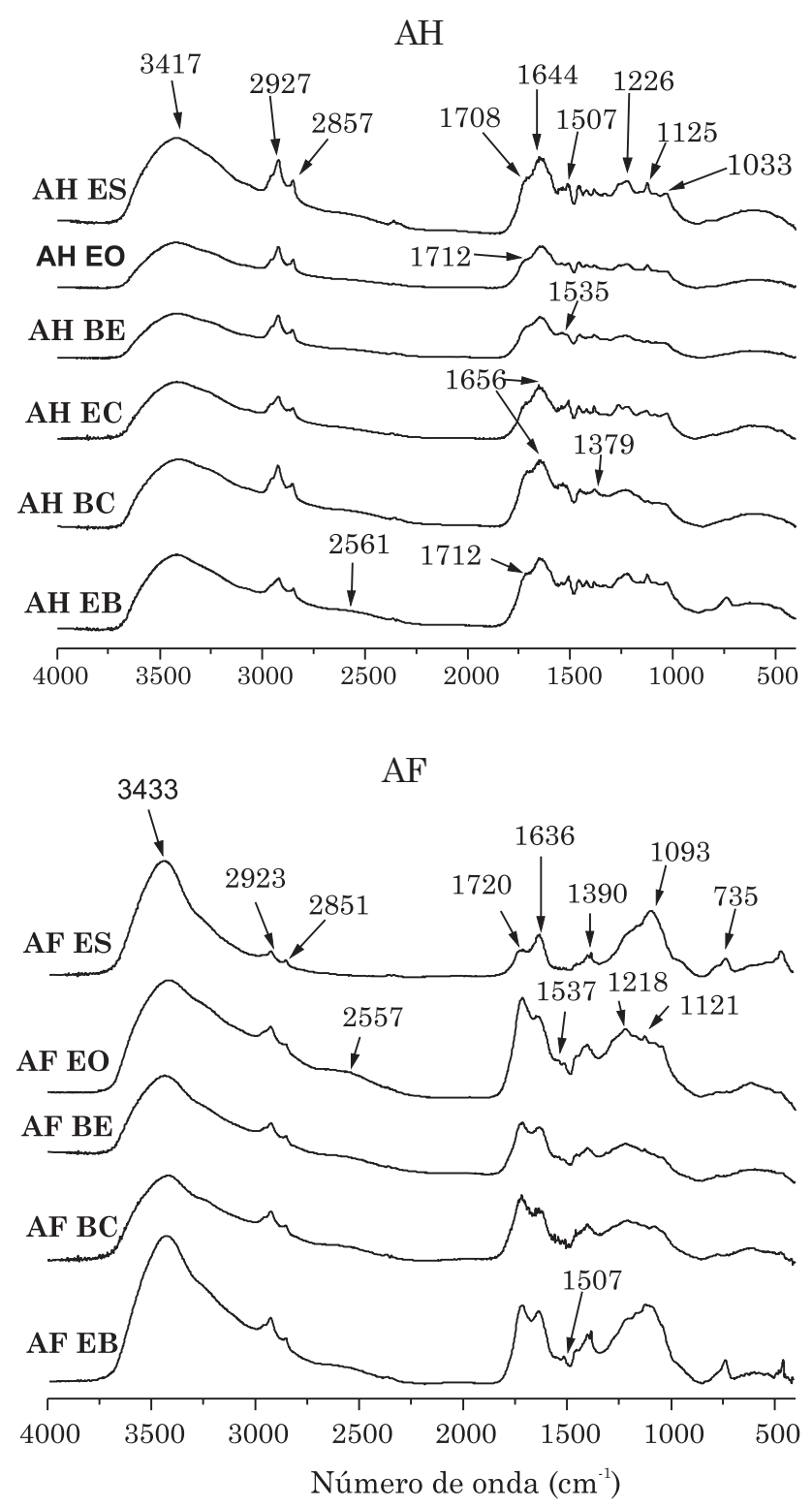

Figura 2. Espectros de FTIR de ácidos húmicos (AH) e de ácidos fúlvicos (AF) de vermicompostos. EB: esterco bovino; de BC: borra de café; EC: esterco de codorna; $\mathrm{BE}$ : borra de erva-mate; EO: esterco ovino; ES: esterco suíno. A amostra de AF de EC não foi analisada em virtude de seu baixo teor no vermicomposto. 
Os espectros de FTIR dos vermicompostos (Figura 3) diferenciam-se principalmente daqueles das $\mathrm{SH}$ pelo não-aparecimento ou pela baixa intensidade da banda de $\mathrm{C}=\mathrm{O}$ de carboxílicos $\left(\approx 1720 \mathrm{~cm}^{-1}\right)$ e pela presença da banda em $1387 \mathrm{~cm}^{-1}$ devida ao estiramento $\mathrm{C}-\mathrm{O}$ de carbonato. $\mathrm{O}$ baixo teor de $\mathrm{C}$ nos

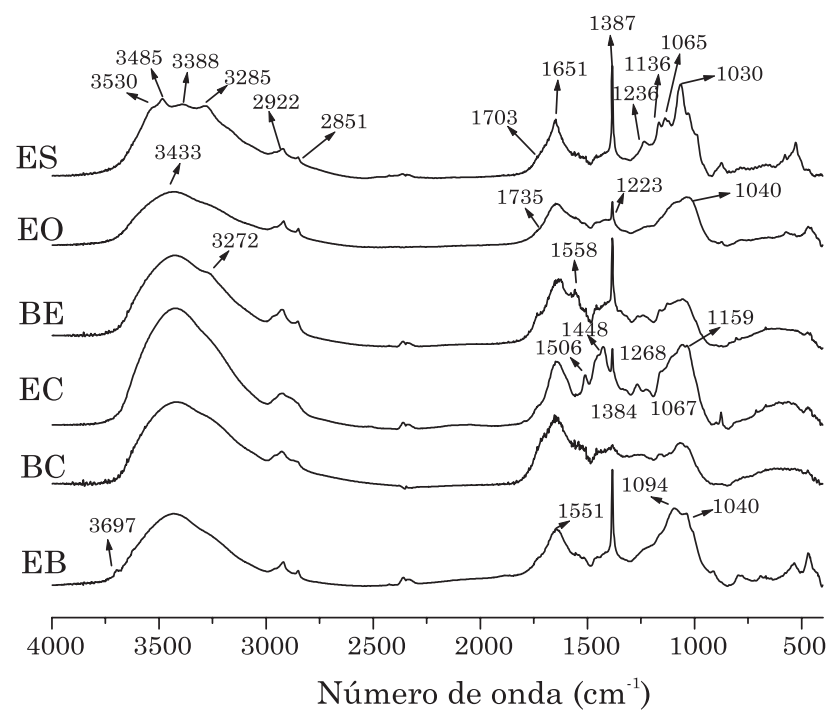

Figura 3. Espectros de FTIR de vermicompostos. EB: esterco bovino; BC: borra de café; EC: esterco de codorna; BE: borra de erva-mate; EO: esterco ovino; ES: esterco suíno. vermicompostos (Quadro1) leva a uma baixa resolução das bandas da fração inorgânica. No entanto, é interessante ressaltar que nos espectros das amostras de origem animal, a banda de absorção em torno de 1150 a $1060 \mathrm{~cm}^{-1}$ atribuída à ligação C-O é proporcionalmente mais acentuada, indicando a presença mais abundante de polissacarídeos nos vermicompostos.

$\mathrm{O}$ valor do índice de aromaticidade $\mathrm{I}_{1630} / \mathrm{I}_{2920}$ determinado a partir dos dados de FTIR é sempre mais elevado nos AF em relação aos AH (Quadro 2), contradizendo os elevados valores de $\mathrm{H} / \mathrm{C}$ que apontam para maior alifaticidade nos AF. Provavelmente, a existência de carboxilatos nessas amostras, que também absorve em torno de $1650 \mathrm{~cm}^{-1}$, leva à superestimação da absorção dos grupos aromáticos. $\mathrm{O}$ elevado teor de O obtido nos AF (Quadro 2) confirma esta hipótese. Os menores valores de aromaticidade foram obtidos nos $\mathrm{AH}$ de origem vegetal.

\section{CONCLUSÕES}

1. Os vermicompostos estudados diferiram quanto à distribuição de substâncias húmicas solúveis $(\mathrm{AH}+\mathrm{AF})$, cujo teor decresceu na seqüência: borra de café $>$ esterco ovino borra de erva-mate esterco bovino > esterco suíno > esterco de codorna.

Quadro 2. Composição elementar, razão C/N, H/C, O/C e índice de aromaticidade FTIR $\left(\mathrm{I}_{1630} / \mathrm{I}_{2920}\right)$ de ácidos húmicos e ácidos fúlvicos de vermicompostos

\begin{tabular}{|c|c|c|c|c|c|c|c|c|}
\hline Amostra & $C^{(1)}$ & $\mathbf{H}^{(1)}$ & $\mathbf{N}^{(1)}$ & $\mathbf{O}^{(2)}$ & $\mathrm{C} / \mathrm{N}$ & $\mathrm{H} / \mathrm{C}^{(3)}$ & $\mathrm{O} / \mathrm{C}^{(3)}$ & $\mathbf{I}_{1630} / \mathbf{I}_{2920}$ \\
\hline & $\mathrm{g} \mathrm{kg}^{-1}$ & & & & & & & \\
\hline \multicolumn{9}{|c|}{ Ácidos húmicos } \\
\hline $\mathrm{EB}$ & 537 & 59,1 & 41,8 & 362 & 12,8 & 1,3 & 0,50 & 1,11 \\
\hline $\mathrm{BC}$ & 550 & 55,0 & 52,5 & 342 & 10,5 & 1,2 & 0,46 & 0,69 \\
\hline $\mathrm{EC}$ & 536 & 58,5 & 44,4 & 361 & 12,1 & 1,3 & 0,50 & 1,12 \\
\hline $\mathrm{BE}$ & 552 & 63,3 & 49,3 & 335 & 11,2 & 1,4 & 0,46 & 0,57 \\
\hline $\mathrm{EO}$ & 533 & 56,3 & 45,8 & 365 & 11,6 & 1,3 & 0,51 & 0,77 \\
\hline ES & 536 & 58,8 & 44,3 & 361 & 12,1 & 1,3 & 0,50 & 0,75 \\
\hline \multicolumn{9}{|c|}{ Ácidos Fúlvicos } \\
\hline $\mathrm{EB}$ & 322 & 71,2 & 22,7 & 546 & 14,2 & 2,7 & 1,43 & 1,05 \\
\hline $\mathrm{BC}$ & 358 & 42,4 & 21,7 & 578 & 16,5 & 1,4 & 1,21 & 7,53 \\
\hline $\mathrm{BE}$ & 397 & 44,6 & 25,7 & 532 & 15,5 & 1,3 & 1,00 & 1,58 \\
\hline $\mathrm{EO}$ & 400 & 44,8 & 30,7 & 524 & 13,0 & 1,3 & 0,98 & 1,38 \\
\hline
\end{tabular}

(1) Base livre de cinzas. ${ }^{(2)}$ Calculado por diferença de $1.000 \mathrm{~g} \mathrm{~kg}{ }^{-1}$. ${ }^{(3)}$ Razões atômicas. EB: esterco bovino; BC: borra de café; EC: esterco de codorna; BE: borra de erva-mate; EO: esterco ovino; ES: esterco suíno. A amostra de AF de EC não foi analisada em virtude de seu baixo teor no vermicomposto. Os dados da composição elementar do AF do ES não foram considerados pois o teor de cinzas deu elevado (84\%). 
2. O grau de maturidade, estimado pelos índices HR e HP, foi superior nos vermicompostos de resíduos de origem vegetal (borra de café e de erva-mate). Os ácidos húmicos desses dois vermicompostos apresentaram menor grau de oxigenação e menor caráter aromático.

3. A composição química dos $\mathrm{AH}$ e dos $\mathrm{AF}$ não refletiu a composição química do vermicomposto.

\section{AGRADECIMENTOS}

Ao CNPq e à FAPERGS, pelas bolsas de Iniciação Científica.

\section{LITERATURA CITADA}

ANTUNES, R.M. Substâncias húmicas originadas de resíduos sólidos agroindustriais compostados. Pelotas, Universidade Federal de Pelotas, 2005. 65p. (Tese de Mestrado)

CASTILHOS, R.M.V.; ANTUNES, R.M.; DICK, D.P.; CASTILHOS, D.D. \& LEAL, O.A. Teor e caracterização de substâncias húmicas em vermicompostos de resíduos agroindustriais. In: CONGRESSO BRASILEIRO DE CIÊNCIA DO SOLO, 31., Gramado, 2007. Anais. Porto Alegre, Universidade Federal do Rio Grande do Sul , 2007. CD-ROM.

CERETTA, C.A.; BAYER, C.; DICK, D.P.; MARTIN-NETO, L. \& COLNAGO, L.A. Métodos espectroscópicos. In: SANTOS, G.A. \& CAMARGO, F.A.O., eds. Fundamentos da matéria orgânica do solo. Porto Alegre, Gêneses, 1999. p.293-336.

CHEFETZ, B.; HATCHER, P.; HADAR, Y. \& CHEN, Y. Chemical and biological characterization of organic matter during composting of municipal solid waste. J. Environ. Qual., 25:776-785, 1996.

CHEN, Y. \& INBAR, Y. Chemical and spectroscopic analyses of organic matter transformations during composting in relation to compost maturity. In: HOITINK, H.A.J. \& KEENER, H.M. INTERNATIONAL COMPOSTING RESEARCH SYMPOSIUM: SCIENCE AND ENGINEERING OF COMPOSTING - DESIGN, ENVIRONMENTAL, MICROBIOLOGICAL AND UTILIZATION ASPECTS, Wooster, 1992. Proceedings. Worthington, Remaissance Publications, 1993. p.551-600.
DICK, D.P.; DICK, Y.P.; DA SILVA, L.F.; ESSIG, C. \& ZORTEA, R.B. Caracterização de produtos de decomposição de lodos orgânicos em diferentes tempos e meio de digestão. R. Bras. Ci. Solo. 21:1-8, 1997.

DICK, D.P.; SANTOS, J.H.Z. \& FERRANTI, E.M. Chemical characterization and infrared spectroscopy of soil organic matter from two Southern Brazilian soils. R.Bras. Ci. Solo, 27:29-39, 2003.

GUERRA, J.G.M. \& SANTOS, G.A. Métodos químicos e físicos. In: SANTOS, G.A. \& CAMARGO, F.A.O., eds. Fundamentos da matéria orgânica do solo. Porto Alegre, Gêneses, 1999. p.267-291.

HUBER, A.C.K. Estudo da mesofouna (ácaros e colêmbolos) e da macrofauna (minhocas) no processo da vermicompostagem. Pelotas, Universidade Federal de Pelotas, 2005. 77p. (Tese de Mestrado)

LEAL, O.A.; ANTUNES, R.M.; CASTILHOS, R.M.V. \& CASTILHOS, D.D. Fracionamento químico da matéria orgânica em resíduos orgânicos e agroindustriais vermicompostados. In: CONGRESSO DE INICIAÇÃO CIENTÍFICA, 15; ENCONTRO DE PÓS-GRADUAÇÃO, 8., Pelotas, 2006. Anais. Pelotas, Universidade Federal de Pelotas, 2006. CD-ROM

SENESI, N.; MIANO, T.M. \& BRUNETI, G. Humic-like substances in organic amendments and effects on native soil humic substances. In: PICCOLO, A. Humic substances in terrestrial ecosystems. Amsterdam, Elsevier, 1996. p.531-595.

STEVENSON, F.J. Humus chemistry: Genesis, composition, reactions. New York, John Wiley \& Sons, 1994. 496p.

TEDESCO, J.M.; GIANELLO, C. \& BISSANI, C.A. Análise de solo, plantas e outros materiais. Porto Alegre, Universidade Federal do Rio Grande do Sul, 1995. 174p. (Boletim Técnico, 5)

TEDESCO, M.J.; SELBACH, P.A.; GIANELLO, C. \& CAMARGO, F.A.O. Resíduos orgânicos no solo e impactos no ambiente. In: SANTOS, G.A. \& CAMARGO, F.A.O., eds. Fundamentos da matéria orgânica do solo. Porto Alegre, Gêneses, 1999. p.159-192.

TIBAU, A.O. Matéria orgânica do solo. In: TIBAU, A.O. .Matéria orgânica e fertilidade do solo. São Paulo, Nobel, 1984. p.49-182. 
